\title{
Co-constructive Logics for Proofs and Refutations
}

\author{
James Trafford \\ University for the Creative Arts, Ashley Road, Epsom, Surrey, KT18 5BE \\ United Kingdom
}

e-mail: jtrafford2@ucreative.ac.uk

\begin{abstract}
:
This paper considers logics which are formally dual to intuitionistic logic in order to investigate a co-constructive logic for proofs and refutations. This is philosophically motivated by a set of problems regarding the nature of constructive truth, and its relation to falsity. It is well known both that intuitionism can not deal constructively with negative information, and that defining falsity by means of intuitionistic negation leads, under widely-held assumptions, to a justification of bivalence. For example, we do not want to equate falsity with the nonexistence of a proof since this would render a statement such as "pi is transcendental" false prior to 1882. In addition, the intuitionist account of negation as shorthand for the derivation of absurdity is inadequate, particularly outside of purely mathematical contexts. To deal with these issues, I investigate the dual of intuitionistic logic, co-intuitionistic logic, as a logic of refutation, alongside intuitionistic logic of proofs. Direct proof and refutation are dual to each other, and are constructive, whilst there also exist syntactic, weak, negations within both logics. In this respect, the logic of refutation is weakly paraconsistent in the sense that it allows for statements for which, neither they, nor their negation, are refuted. I provide a proof theory for the co-constructive logic, a formal dualizing map between the logics, and a Kripke-style semantics. This is given an intuitive philosophical rendering in a re-interpretation of Kolmogorov's logic of problems.
\end{abstract}

Keywords: constructivism, paraconsistency, intuitionistic logic, co-intuitionistic logic, negation.

\section{Constructive Truth and Falsity}

\subsection{Constructivism and undetermined statements}

It is not altogether simple to pin down precisely what defines a logic as constructive. As is known, there are classically valid formulas that do not admit constructive proofs. More pressing, however, is the non-determinism that results from this lack of constructivity due to the appearance of multiple (disjunctive) succedents in typical presentations of classical logic (e.g Gentzen's $L K$ ). Constructive logics, by the disjunction property, ensure that a (cut-free) proof of $\alpha \vee \beta$ (where both $\alpha$ and $\beta$ have single, cut-free proofs), must be a proof of one of the disjuncts ${ }^{1}$. Classically this breaks down since, by contraction, there may exist a disjunction $\alpha \vee \beta$ without any means of deciding which formula is proved. So, one may require that a constructive logic has the disjunction property, or some analogue of it. 
This is a decent enough technical requirement, but it does not seem to get to the heart of constructivism. Rather, a key requirement on constructivism is typically that it admits a constructive account of truth, such for a proposition $\alpha$ to be "true" consists in there existing a constructive proof of $\alpha$, and that, once true it remains so (monotonicity). However, pinning exactly what this requires is, notoriously tricky, resulting in a bifurcation of the understanding of what is meant by proof in the literature [17], [23], [27] into:

- Actualism (there actually exists a proof of $\alpha$ )

- Potentialism ( $\alpha$ is potentially provable $)^{2}$.

Actualism is a tensed, time-bound, notion of proof given in a specific moment, and is typically thought to have been held by Brouwer and Heyting, see e.g. [13]. Potentialism is, rather, an objective notion of proof, typically held by Prawitz [21], and by Dummett (at certain points [9]). The open question here is whether or not for $\alpha$ to count as constructively true, actual possession of a proof of $\alpha$ is required of an agent, or merely an effectively decidable possible proof. The latter, (as pointed out in e.g. [16, pp.84-85]) seems to require commitment to an objective realm of propositions that ultimately faces similar objections to the classical account, leading to an "inert platonism of proofs" [6]. So, potentialism leads to a problematic account of truth regarding propositions that is both tenseless and independent of subjective understanding:

[This] pays too much tribute to a "platonician" conception of mathematical inference: according to this conception, far from being deduced or extracted by ourselves, the consequences of an hypothesis follow from it by themselves, or rather in virtue of the existence of certain objects that it is none of our responsibility to conceive, or to make up, but only to discern; these objects may be likened to documents namely of documents already written, perhaps never read by anybody yet, but in which we could not fail to recognize, were we to become acquainted with them [...] By identifying proofs with sequences of formulas or, more generally, with objects that are independent from us, one almost unavoidably reduces the activity of justification to a scanning and control process that requires no cognitive or physical particular resource, and that consequently could not be affected by the limits of the cognitive capacities of the agents [5].

Potentialism, then, would not seem to adequately capture what is "constructive" regarding constructivism, which awards a key role to the actions of agents involved in the construction of a proof. But, the actualist position, depending upon how it is construed, may not alleviate these concerns. Insofar as the existence of a proof is understood in terms of a proof as "object", as, for example, Hilbert-style calculus seems to require, proofs will also be reduced to a form of ratification, where proofs are unaffected by our interaction with them.

To this end, we will work with a traditional, actualist, conception of proof which understands proofs in terms of "act" rather than object. It is clear, on Brouwer's account, for example, that something occurs whenever an agent comes to have an effective decision procedure for a proposition $\alpha$ : law of excluded middle becomes valid for $\alpha$ (e.g. [2]). Perhaps then, what is key to considering constructivism, is that formulas such as $\alpha$ may be reasoned with, considered, and so on, without the presumption of the existence of such a procedure, and, moreover, without even understanding $\alpha$ to be a proposition, at least insofar as a proposition is supposed to be semantically evaluable, and so on. If this is correct, then at the heart of constructivism, is that it allows for the existence of undecidability in the structure of syntax itself, and that our actions can render what is undecidable now to become decidable at some future moment. In this vein, we may follow Dubucs and Marion [6], who consider proof as "act", rather than "object": 
We propose that one distinguishes between two different notions of proof, namely those of proof as 'object' and as 'act'. According to the first conception, a proof is something like an assemblage of strings of symbols satisfying such and such property. From the second, more dynamic, conception, a proof is a process whose result may be represented or described by means of linguistic symbols.

This interpretation is appropriate to the typical BHK interpretation of logical connectives, which takes the justification of the assertion of a complex proposition to consist in the justification of its immediate constituents. This allows for a conception of proofs as acts, for which the BHK interpretation provides an account of their complex composition. In fact, this is clearer in Kolmogorov's [14] interpretation than even Brouwer's, which argues that constructive logic has to do "not with theoretical propositions but, on the contrary, problems". ${ }^{3}$ In this setting, it is simple to see that what is central to constructivity is that it allows us to deal with undetermined formulas (or problems):

Undetermined: $(\exists \alpha) \alpha \mid-$ and $\neg \alpha \mid-$.

In terms of a standard Kripke structure, $\mathrm{M}$ as a triple $\mathrm{M}=_{d f}\left(\mathrm{~S}, \leq_{+}, \|-^{+}\right)$, where $\mathrm{S}$ is a collection of "states", or "stages", of reasoning, $\leq_{+}$is a pre-order on $S$, and $\|^{+}$is a "forcing relation" between formulas of a language and elements of $\mathrm{S}$. Let $s_{i}$ indicate a stage of the construction, then, we have:

Undetermined: $(\exists \alpha),\left(\exists s_{i} \in \mathrm{S}\right), s_{i} \|+^{+} \alpha \quad$ and $\quad s_{i} \| f^{+} \neg \alpha$.

\subsection{Asymmetry of truth and falsity}

But, what is the status of undetermined formulas? There is an asymmetry between proof (and truth) and refutation (and falsity) in intuitionistic logic, which is particularly problematic from the point of view of constructive falsity. Undetermined formulas might simply be taken to be false, so that falsity is equated with non-truth. But, it does not seem right to say, of some formula $\alpha$ which is not provable at a stage $s_{1}$ but may become provable at a later stage $s_{2}$, that it is false, and then becomes true. Instead, falsity is typically equated with a reduction to a contradiction (this is the case in Heyting [13], and also Kolmogorov [14]), so that $\alpha$ is false whenever $\neg \alpha$ is true. This, too, is not unproblematic. As Shramko et al. [27] point out, if we attempt to formalize the definition of falsity in terms of negation, then we are led to a reliance, not only on a syntactic feature (negation), but also on truth, so there is a lack of independence of falsity. Moreover, as Dummett [7] is aware, this, together with potentialism is constructively suspect:

There is a well-known difficulty about thinking of mathematical proofs [...] as existing independently of our hitting on them, which insisting that they are proofs we are capable of grasping or of giving fails to resolve. Namely, it is hard to see how the equation of the falsity of a statement (the truth of its negation) with the non-existence of a proof or verification can be resisted: but, then, it is equally hard to see how, on this conception of the existence of proofs, we can resist supposing that a proof of a given statement either exists or fails to exist. We shall then have driven ourselves into a realist position, with a justification of bivalence. If we refuse to identify falsity with the non-existence of a proof, we shall be little better off, because we shall find it 
hard to resist concluding that there are statements which are determinately neither true nor false $[\ldots]\left[6\right.$, p. 285]. ${ }^{4}$

Kolmogorov provides us with a possible route around this issue by allowing that undetermined formulas are problems, not propositions. As such, these are just not the sort of thing that can be understood to be true or false. Rather, at the level of syntax, we have the capacity to deal with formulas that are undetermined, and, it is our action on syntax through which propositions become determined. This closely follows Martin-Löf's [16] argument that: 'A proof is, not an object, but an act $[. .$.$] , and the act is primarily the act as it is being performed, only secondarily, and irrevocably,$ does it become the act that has been performed.' In Kolmogorov's terms, this secondary object is the "solution" to a problem, and this is a proposition, and so truth-evaluable. An unsolved problem is not false (a problem is not the sort of thing that could be false), rather it is unproved, and so, nonaccepted. Of course, this is to say that this state of a formula $\alpha$ must be non-monotonic in the sense that it must be possible to move from an unproved formula to a proved solution (but not back again). In other words, whilst the logic of problems is constructive, for solutions (propositions), law of excluded middle holds, so determination for a formula has a clearly link to truth-evaluability. We will return to this later.

Even if this is plausible, however, we are still left with two (connected) issues: one has to do with negation, and the other the asymmetry of truth. As is known, intuitionistic logic is incapable of refuting formulas directly. Reductio ad absurdum is capable only of conditionally proving negative statements, since $\neg \neg \alpha \rightarrow \alpha$ does not hold in general. So, even if we can show that $\neg \alpha$ leads us to a contradiction, we are not in a position to prove $\alpha$. In addition, a negative answer to a problem will not, in general, return a solution. For example, if we answer "is $\alpha$ provable (at some stage $\left.s_{i}\right)$ ?" negatively, this "no" represents something like a withdrawal that does not involve any commitment to a solution of $\alpha$ or $\neg \alpha$. But, this "no" can not be what is expressed by intuitionistic negation, since that is supposed to record commitment to $\neg \alpha$, whose truth is constructive (i.e. when we have a proof of $\neg \alpha$, this can not change over progressive stages of reasoning). This is problematic, since we do not, for example, want to say even of a mathematical statement that it is false if it is not proved, since then a statement such as " $\pi$ is transcendental" would be deemed false prior to 1882 . Then, to deal with statements which are provable, but not yet proved, requires a weaker than intuitionistic negation:

Our reluctance to say that $\pi$ was not transcendental before 1882 , or, more significantly, to construe mathematical statements as significantly tensed, is not merely a lingering effect of platonistic misconceptions; it is, rather, because to speak in this way would be to admit into mathematical statements a non-intuitionistic form of negation, as will be apparent if one attempts to assign a truth-value to "pi is not algebraic" considered as a statement made in 1881. This is not because the "not" which occurs in "...is not true" or "...was not true" is non-constructive: we may reasonably view it as decidable whether or not a given statement has been proved at a given time. But, though constructive, this is an empirical type of negation, not the negation that occurs in statements of intuitionistic mathematics. The latter relates to the impossibility of ever carrying out a construction of some fixed type, the former to the outcome, at variable times, of some fixed observation or inquiry [8, p. 337]. 
Similarly, standard intuitionistic negation is too strong to express statements such that Goldbach's conjecture is undecided at present. What would be required, instead, is a weaker negation, which expresses that the statement lacks a proof at the present stage of reasoning. This weaker negation is precisely the "not" in the statements that Dummett analyses insofar as it records non-provability at a stage of reasoning. One of the properties of this weaker negation must be that, at some future stage of reasoning, the statement may be proved or refuted (and so, the problem solved). It seems, given the above arguments, that this is a key feature of any constructivism, and so, without it, we have an expressive inadequacy regarding the ability to express undeterminedness.

In this sense, typical construals of constructive negation appear as both too weak and too strong. On the one hand, it is not possible to directly establish that some formula $\alpha$ is refuted, rather, it is possible only to conditionally prove that, from assuming $\alpha$, we can derive a contradiction. On the other, we seem to require a kind of "weak negation" where some potential proof of $\alpha$ does not go through, and so, expresses that no proof of $\alpha$ exists at that stage of reasoning, though without also precluding there being a proof of $\alpha$ or $\neg \alpha$ at some later stage. The former issue has to do with a symmetrical means by which it would be possible to directly refute some formula $\alpha$; the second, with a means of recording a "withdrawal" or state of nonacceptability for $\alpha$, which does not preclude later proof or refutation.

Consider the former issue in relation to Kolmogorov's interpretation. There, we have for some problem $\alpha$, the asymmetric pair of questions: "is $\alpha$ provable?"; "is $\alpha$ reducible to a contradiction?". If, instead, we conceive of refutation as counterpart to proof, this would lead us to the symmetric pair: "is $\alpha$ provable?"; “is $\alpha$ refutable?". Whilst not strictly belonging to intuitionistic logic, this may be perfectly constructive. Indeed, Dummett [8] points out that it may be required outside of anything but a purely mathematical context:

[A] proof of the negation of any arbitrary statement then consists of an effective method for transforming any proof of that statement into a proof of some false numerical equation. Such an explanation relies on the underlying presumption that, given a proof of a false numerical equation, we can construct a proof of any statement whatsoever. It is not obvious that, when we extend these conceptions to empirical statements, there exists any class of decidable atomic statements for which a similar presumption holds good; and it is therefore not obvious that we have, for the general case, any similar uniform way of explaining negation for arbitrary statements. It would therefore remain well within the spirit of a theory of meaning of this type that we should regard the meaning of each statement as being given by the simultaneous provision of a means for recognizing a verification of it and a means for recognizing a falsification of it, where the only general requirement is that these should be specified in such a way as to make it impossible for any statement to be both verified and falsified [7, p. 71].

Should we translate the stipulation into a BHK-style clause for negation, so that the negation of $\alpha$ is verified whenever $\alpha$ is falsified (and vice-versa), then this is analogous to Nelson's strong negation [19], and discussed in a similar context in [20].

In the following, we take a different tack, and consider the dual logic to intuitionistic logic, co-intuitionistic logic as a logic of refutation. This, I suggest, solves the first issue regarding "strong" negation, since this will be encoded in direct refutation. The second issue, regarding "weak" negation will be solved through consideration of the interaction between the two logics. 


\section{Constructive Co-intuitionistic Logic}

In this section we provide an interpretation of co-intuitionistic logic as a constructive logic of refutation. To highlight the constructive nature of this logic, we note that the dual of "Undetermined" holds:

$$
\text { Inconsistent: }(\exists \alpha) \alpha-\mid \text { and } \neg \alpha-\text { (where }-\mid \text { indicates a "refutation" relation). }
$$

Again, this may be understood constructively, as saying that there exists some formula $\alpha$, for which neither $\alpha$, nor its negation are refuted. This is not to say that $\alpha, \neg \alpha$ are considered "true", rather they too are undetermined in the sense that they are not yet refuted; they are "nonrejectable". Again, this can be expressed in terms of a Kripke structure for refutation, M as a triple $\mathrm{M}=_{d f}\left(\mathrm{~S}, \leq_{-}, \|-^{-}\right)$, where $\mathrm{S}$ is a collection of "states", or "stages", of reasoning, $\leq_{-}$is a pre-order on $S$, and $\|^{-}$is a refutation "forcing relation" between formulas of a language and elements of $S$. Let $s_{i}$ indicate a stage of the construction, then, we have:

Inconsistent: $(\exists \alpha),\left(\exists s_{i} \in \mathrm{S}\right), s_{i} \| t^{-} \alpha$ and $s_{i} \| t^{-} \neg \alpha$.

Here, there are problems which are undetermined, and so inconsistent (since neither are refuted). A refutation provides a way of determining a proposition, and we will show that the dual of the disjunction property, the conjunction property, ensures that law of non-contradiction holds for determined propositions. Understood this way, a paraconsistent logic of refutations admits constructivity just as an intuitionistic logic of proofs.

This is more perspicuous if we tackle this proof-theoretically.

Definition 1 (Languages $\left.S, S^{d}\right)$. Over a denumerable set of atomic formulas, the languages for intuitionistic and co-intuitionistic logic are defined in Backus-Naur form ( $\alpha$ is any atomic formula): ${ }^{5}$

$$
\begin{aligned}
S \beta: & =\left[\alpha\left|\left(\neg_{I} \beta\right)\right|(\beta \wedge \beta)|(\beta \vee \beta)|(\beta \Rightarrow \beta)\right] \\
S^{d} \beta & :=\left[\alpha\left|\left(\neg_{C} \beta\right)\right|(\beta \wedge \beta)|(\beta \vee \beta)|(\beta \Leftarrow \beta)\right]
\end{aligned}
$$

Here, $\neg_{I}$ and $\neg_{C}$ denote the negations of intuitionistic and co-intuitionistic negation, respectively, and $\Rightarrow$ and $\Leftarrow$ denote implication and co-implication, respectively. ${ }^{6}$

The usual construction of a deductive (or inference) structure involves their being a proof of some formula $\alpha$, from a (possibly empty) set of formulas, $\Gamma$. Typically, we think of this as saying that $\alpha$ may be asserted in the context of $\Gamma$. So, intuitionism provides an account of the assertibility conditions for a set of formulas, with the general condition that a formula $\alpha$ is assertible whenever there is a proof of $\alpha$. Here, we are also considering denial as the dual of assertion, and so we require an account of the deniability conditions for a set of formulas, perhaps with the general condition that a formula $\alpha$ is deniable whenever there is a refutation of $\alpha$. To this end, we will 
consider a co-intuitionistic structure (as defined in [35]) as a refutation system, following the direction of [28], [29], [30]. The meaning of a sequent of the form $\Gamma \mid{ }_{I} \alpha$ is that, whenever there is a proof of $\Gamma$, there is also a proof of $\alpha$ (in intuitionistic logic). We also introduce ${ }_{C}-\mid$, where the meaning of a sequent of the form $\Gamma_{C}-\mid \alpha$ is that, under the assumption that $\Gamma$ is refuted, $\alpha$ is refuted also. Just as $\varnothing \mid{ }_{I} \alpha$ indicates that $\alpha$ is a theorem of an intuitionistic proof-theory, $\varnothing_{C}-\alpha$ indicates that $\alpha$ is a "theorem" of a co-intuitionistic refutation-theory.

Remark 1. Intuitionistically, implication internalizes the metalinguistic deducibility relation by means of the deduction theorem: $\Gamma\left|{ }_{I} \alpha \leftrightarrow\right|{ }_{L} \bigcap \Gamma \Rightarrow \alpha$. In co-intuitionistic logic, we can read co-implication as exactly dual: $\Gamma_{C}-\alpha \leftrightarrow{ }_{c}-\alpha \Leftarrow \bigcap \Gamma$.

With these in hand, we can define an inference structure $L$, and its dual, $L^{d}$, where $L$ is understood to be a typical proof-structure, and $L^{d}$, a structure for refutation.

Definition 2 (Inference structure). Define an inference structure $L$ as an ordered pair, $\left\langle S, \mid-_{L}\right\rangle$, where $S$ is as above, and $\left.\right|_{L}$ is a binary derivability relation between a subset of formulas of $S$ (denoted $(\Gamma, \Delta))$, and formulas of $S,($ denoted $(\alpha, \beta))$, so $\mid-{ }_{L} \subseteq \mathrm{P}(S) \times S .{ }^{7}$ Say that $\mid{ }_{L}$ is normal when it is reflexive, transitive, monotonic and finitary. A sequent in $L$ is an ordered pair $\langle\Gamma, \alpha\rangle$ where $\Gamma$ is a set of formulas and $\alpha$ a single formula (and $\Gamma \cup\{\alpha\}$ ).

The dual notion of refutation is now simple to define.

Definition 3 (Sequent duality). The dual of $\Gamma \mid{ }_{L} \alpha$ is $\Gamma_{L^{d}}^{d}-\mid \alpha^{d}$ (for any $\Gamma \subseteq S, \alpha \in S$ and $\left.\Gamma^{d} \subseteq S^{d}, \alpha^{d} \in S^{d}\right)$.

Definition 4 (Dual inference structure). Define a dual inference structure $L^{d}$ as an ordered pair, $\left\langle S^{d},{ }_{L^{d}}-\right\rangle$, where $S^{d}$ is as above, and ${ }_{L^{d}}-\mid$ is a binary derivability relation between a subset of formulas of $S^{d}$ and formulas of $S^{d}$, so ${ }_{L^{d}}-\mid \subseteq \mathrm{P}\left(S^{d}\right) \times S^{d}$. Again, say that ${ }_{L^{d}}-$ is normal when it is reflexive, transitive, monotonic and finitary. A sequent in $L^{d}$ is an ordered pair $\langle\Gamma, \alpha\rangle$ where $\Gamma$ is a set of formulas and $\alpha$ a single formula (and $\Gamma \cup\{\alpha\}$ ), bearing in mind that these belong to $S^{d}$.

In foregrounding the notion of refutation, we are interested in the conditions under which $\alpha$ is refutable given a set of (denied) assumptions $\Gamma$, thus allowing for a non-trivial account of the deniability of $\alpha$ in the context of $\Gamma$. Of course, if we were working in classical logic, then $L$ and $L^{d}$ may be dealt with utilizing a multiple-conclusion structure such as Gentzen's $L K$, which is selfdual. ${ }^{8}$ But, here, we are interested in the non-self-dual, but, nonetheless, dual structures, intuitionistic logic and co-intuitionistic logic. We briefly sketch the details, closely following the presentation found in [35]. I assume familiarity with the system $L J$, denoted $I$, which is obtained 
through the restriction of symmetric sequents to sequents in which a multiset of formulas may appear in the antecedent, but (at most) a single formula in the consequent. Analogously, the system $L D J$ is usually obtained through a restriction so that (at most) a single formula may appear in the antecedent. However, in distinction to the typical presentation in, e.g. [35], due to the way in which we are reading refutation sequents $\Gamma_{C}^{d}-\mid \alpha^{d}$, this will amount to a restriction to singularity on the right.

Definition 5 (The system $L D J$, denoted $C$ ). Since all formulas are constructed from $S^{d}, I$ will drop the superscript in what follows, apart from the identity axiom.

$$
\begin{aligned}
& \alpha^{d}{ }_{C}-\alpha^{d} \text { (Id) } \\
& \frac{\Delta_{C}-\mid}{\Delta_{C}-\alpha}(\text { Thin- } R) \quad \frac{\Delta_{C}-\beta}{\Delta, \alpha_{C}-\beta}(\text { Thin- L }) \\
& \frac{\Delta, \alpha, \alpha_{C}-\beta}{\Delta, \alpha_{C}-\beta} \text { (Cont) } \\
& \frac{\Delta, \alpha, \sigma, \Theta_{C}-\beta}{\Delta, \sigma, \alpha, \Theta_{C}-\beta}(\text { Int }) \\
& \frac{\Delta, \alpha_{C}-\beta \Gamma_{C}-\beta}{\Delta, \Gamma_{C}-\beta}(\mathrm{Cut}) \\
& \frac{\Delta_{C}-\alpha}{\Delta_{C}-\alpha \wedge \beta}\left(\wedge R_{1}\right) \\
& \frac{\Delta_{C}-\beta}{\Delta_{C}-\alpha \wedge \beta}\left(\wedge R_{2}\right) \\
& \frac{\Delta, \alpha_{C}-\sigma \quad \Delta, \beta_{C}-\sigma}{\Delta, \alpha \wedge \beta_{C}-\sigma}(\wedge L) \\
& \frac{\Delta_{C}-\alpha \Delta_{C}-\beta}{\Delta_{C}-\alpha \vee \beta}(\vee R) \\
& \frac{\Delta, \alpha_{C}-\sigma}{\Delta, \alpha \vee \beta_{C}-\sigma}\left(\vee L_{1}\right) \\
& \frac{\Delta, \beta_{C}-\sigma}{\Delta, \alpha \vee \beta_{C}-\sigma}\left(\vee L_{2}\right) \\
& \frac{\Delta, \beta_{C}-\alpha}{\Delta_{C}-\alpha \Leftarrow \beta} \quad(\Leftarrow R) \\
& \frac{\Delta, \alpha_{C}-\sigma \quad \Gamma_{C}-\beta}{\Delta, \Gamma, \alpha \Leftarrow \beta_{C}-\sigma}(\Leftarrow L) \\
& \frac{\Delta, \alpha{ }_{C}-\mid}{\Delta_{C}-\neg_{C} \alpha}\left(\neg_{C} R\right) \\
& \frac{\Delta_{C}-\alpha}{\Delta, \neg_{C} \alpha{ }_{C}-\mid}\left(\neg_{C} L\right)
\end{aligned}
$$

Theorem 1. The rule Cut is eliminable from the system $C$. 
Proof. The proof is routine and can be found in [35].

As is known, Cut is eliminable from the system $I$.

Corollary 1. The system $C$ has the subformula property and is consistent.

The constructivity of co-Intuitionistic logic (understood as a logic of refutation) is closely related to this conjunction property:

$$
{ }_{C}-\alpha \wedge \beta \text { iff }{ }_{C}-\mid \alpha \text { or }{ }_{C}-\beta \text {. }
$$

Theorem 2. Co-Intuitionistic logic (as refutation) has the conjunction property.

Proof. The proof follows the typical one given for the disjunction property in $I$. Say that there exists a cut-free proof of ${ }_{C}-\alpha \wedge \beta$, then, since it is not an axiom, the proof must end with a rule. This must be one of either $\wedge R_{1}$ or $\wedge R_{2}$, and so from either ${ }_{C}-\mid \alpha$ or ${ }_{C}-\beta$. The other direction is obvious.

We can now precisely define the duality between the intuitionistic and co-intuitionistic inference structures.

Definition 6. We can define a mapping from $S$ to $S^{d}$ (and back) $(-)^{d}: S \leftrightarrow S^{d}$ :

$$
\begin{gathered}
\alpha^{d}: \alpha \text { for atomic } \alpha \\
(\alpha \wedge \beta)^{d}: \alpha^{d} \vee \beta^{d} \\
(\alpha \vee \beta)^{d}: \alpha^{d} \wedge \beta^{d} \\
\left(\neg_{l} \alpha\right)^{d}: \neg_{C}(\alpha)^{d} \\
(\alpha \Rightarrow \beta)^{d}: \beta^{d} \Leftarrow \alpha^{d} \\
\left\{\alpha_{1} \ldots \alpha_{n}\right\}^{d}:\left\{\alpha_{1}^{d} \ldots \alpha_{n}^{d}\right\} \\
\left(\varnothing \mid-{ }_{L} \alpha\right)^{d}: \varnothing_{L^{d}}-\mid \alpha^{d} \\
\left(\Gamma \mid-{ }_{L} \alpha\right)^{d}: \Gamma_{L^{d}}^{d}-\mid \alpha^{d}
\end{gathered}
$$


Here, $(-)^{d}$ is a mapping that is involutive, and without fixed points, operating as a means of expressing the duality relationship between proofs and refutations. ${ }^{9}$

The above can be interpreted in a "falsity-preserving" Kripke-structure as follows.

Definition 7 (Co-intuitionistic Kripke-structure). We define a refutation Kripke structure, $\mathrm{M}$ as a triple $\mathrm{M}=_{d f}\left(\mathrm{~S}, \leq_{-}, \|-^{-}\right)$, where $\mathrm{S}$ is a collection of "states", or "stages", of reasoning, $\leq_{-}$ is a pre-order on $\mathrm{S}$, and $\|_{-}^{-}$is a refutation "forcing relation" between formulas of a language and elements of $\mathrm{S}$. Let $s_{i}$ indicate a stage of the construction where $i, 1 \leq_{-} i \leq n$. Then, at any stage, $s_{i}$, we define: $V^{-}(\alpha)=\left\{\alpha \in S:{s_{i}}^{-} \alpha\right\}$.

For compound formulas, the following conditions hold:

$$
\begin{aligned}
& {[\wedge] s_{i} \|-^{-}(\alpha \wedge \beta) \quad \text { iff } \quad \alpha \in\left(V^{-}, s_{i}\right) \quad \text { or } \quad \beta \in\left(V^{-}, s_{i}\right)} \\
& {[\vee] s_{i} \|-^{-}(\alpha \vee \beta) \quad \text { iff } \quad \alpha \in\left(V^{-}, s_{i}\right) \quad \text { and } \quad \beta \in\left(V^{-}, s_{i}\right)} \\
& {\left[\neg_{C}\right] s_{i} \|-^{-}\left(\neg_{C} \alpha\right) \quad \text { iff } \quad \forall s_{i}^{\prime} \text { and } s_{i} \leq_{-} s_{i}^{\prime}, \alpha \notin\left(V^{-}, s_{i}^{\prime}\right)} \\
& {[\Leftarrow] s_{i} \|-^{-}(\alpha \Leftarrow \beta) \quad \text { iff } \quad \exists s_{i}^{\prime} \text { and } s_{i} \leq_{-} s_{i}^{\prime}, \alpha \in\left(V^{-}, s_{i}^{\prime}\right) \text { or } \beta \notin\left(V^{-}, s_{i}^{\prime}\right) .}
\end{aligned}
$$

Remark 2. Note that this interpretation is atypical in relation to other interpretations of coIntuitionism since we take are considering it from the point of view of constructivity over $V^{-}$. So, the above clauses tell us that, for example, a refutation of $(\alpha \wedge \beta)$ consists of a refutation of $\alpha$, or a refutation of $\beta$. Most interestingly, perhaps, is that, in typical constructions of co-intuitionistic logics (and many other paraconsistent logics), there is no operator definable that is an analogue to modus ponens. As is clear, this is not the case in our refutation interpretation.

Lemma 1. The co-intuitionistic logic is sound and complete w.r.t the Kripke structure for refutation.

Proof. This is easily checked by simply dualizing the standard approach to the Kripke structure for intuitionistic logic.

Algebraically, a semantics can easily be given in a Brouwerian (sometimes called coHeyting) algebra, which is the dual to Heyting algebra, e.g. [11]. Moreover, [17, §11] shows that co-intuitionistic logic is the internal logic of a complement topos, which is dual to a topos (whose internal logic is intuitionist). The former is a category, with terminal object 1 , defined as an object $\Omega$ with an arrow $F: 1 \rightarrow \Omega$, and where $F$ is dual to $\mathrm{T}$ in an ordinary topos. ${ }^{10}$ This suggests that co-intuitionistic logic, interpreted as a constructive logic of refutations, may well be valuable in its own right.

\subsection{Negation, again}


Let us consider the interpretations of negation for intuitionistic and co-intuitionistic logic, respectively, in order to determine the relation between the two:

$$
\begin{aligned}
& {\left[\neg_{I}\right] s_{i} \|-^{+}\left(\neg_{I} \alpha\right) \quad \text { iff } \quad \forall s_{i}^{\prime} \text { and } s_{i} \leq_{-} s_{i}^{\prime}, \alpha \notin\left(V^{+}, s_{i}^{\prime}\right)} \\
& {\left[\neg_{C}\right] s_{i} \|-^{-}\left(\neg_{C} \alpha\right) \quad \text { iff } \quad \forall s_{i}^{\prime} \text { and } s_{i} \leq_{-} s_{i}^{\prime}, \alpha \notin\left(V^{-}, s_{i}^{\prime}\right)}
\end{aligned}
$$

Both of the above correspond to the idea that negation can be given an intuitive reading in terms of the respective implications of the two structures. Just as intuitionistic negation is typically defined as $\neg_{I} \alpha: \alpha \rightarrow \perp$, where $\perp$ is a contradictory statement, so co-intuitionistic negation is typically defined as $\neg_{C} \alpha: \mathrm{T} \leftarrow \alpha$, where $\mathrm{T}$ is a tautologous statement. This reading of the respective negations ensures that $\left(\neg_{I} \alpha\right)$ can never be proved, and $\neg_{C} \alpha$, can never be refuted. So, one, perhaps obvious, suggestion would be to tie the two logics together by means of negation, so that we define the two negations along the lines suggested by Dummett:

$$
\begin{aligned}
& {\left[\neg_{I}\right] s_{i} \|-^{+}\left(\neg_{I} \alpha\right) \quad \text { iff } \quad \forall s_{i}^{\prime} \text { and } s_{i} \leq_{-} s_{i}^{\prime}, \alpha \in\left(V^{-}, s_{i}^{\prime}\right)} \\
& {\left[\neg_{C}\right] s_{i} \|-^{-}\left(\neg_{C} \alpha\right) \quad \text { iff } \quad \forall s_{i}^{\prime} \text { and } s_{i} \leq_{-} s_{i}^{\prime}, \alpha \in\left(V^{+}, s_{i}^{\prime}\right)}
\end{aligned}
$$

That is, the negations of the two structures simply tells us that the negation of a proof intuitionistically, is equivalent to a refutation, co-intuitionistically. ${ }^{11}$ But, simply adding constructive refutation to intuitionistic logic, however, would do nothing to alleviate the issue discussed above regarding "weak" negation. In general, if we accept the metalinguistic restriction that no statement can be both proved and refuted, no syntactic negation need even be involved in that relationship. Moreover, far from disappearing, the problem of asymmetry will now rear its head again, since the idea of "non-acceptability" for a formula, which corresponds to "not yet determined", has no counterpart for "non-rejectability" given that refutation is now a direct notion. What seems to be required, is an interpretation of a syntactic negation operator which, attached to a formula $\alpha$ expresses the idea that a proof of $\alpha$ "does not go through", and so, leaves $\alpha$ undetermined. ${ }^{12}$ This appears perfectly possible, now that we have expanded our original language with the dual language for co-intuitionistic logic.

Further still, as mentioned above, the typical intuitionistic negation defined by introducing a constant, $\perp$, whilst fairly simple to interpret mathematically (as an arithmetic absurdity, say), is far trickier in general. In fact, as pointed out in [3], even in the domain of arithmetic, there appears to be an ineliminable (and vicious) circularity in the typical definition of negation as $\alpha \rightarrow 0=1{ }^{13}$ Similarly, Tennant [32] argues against the view that proofs of negation can be understood as a proof of a false sentence (such as $\perp$ ). Instead, Tennant takes a view similar to Brouwer's, that negation can be understood in terms of a "construction that no longer goes", suggesting that negation indicates a "dead-end" of a construction. The difficulty with these attempts to construe the "correct" definition of constructive negation lies with the fact that the desiderata pulls us in different directions. But, since we are now allowing for direct refutation to exist within the co-intuitionistic structure, we might consider, in addition, a pair of "weak negations", which are defined purely by means of proof-theoretic constraints upon contrariety and sub-contrariety, respectively. ${ }^{14}$ 
These "weak" negation can then be interpreted as:

$$
\begin{aligned}
& {\left[\neg_{I}\right] s_{i} \|-^{+}\left(\neg_{I} \alpha\right) \quad \text { iff } \quad \alpha \notin\left(V^{+}, s_{i}\right)} \\
& {\left[\neg_{C}\right] s_{i} \|-^{-}\left(\neg_{C} \alpha\right) \quad \text { iff } \quad \alpha \notin\left(V^{-}, s_{i}^{\prime}\right)}
\end{aligned}
$$

Of course, the standard negation as derivation of a contradiction (or tautology) does this, but this "reaches" across stages to index the impossibility of $\alpha$ being forced at any stage. The weaker negation allows that $\alpha$ remains an open problem, and so can make sense of the status of claims such as " $\pi$ is transcendental" prior to 1882 . Of course, the status of weakly negated formulas can not then be monotonic, but, as $[29, \S 2.4]$ point out, adding such an operator to standard intuitionistic logic would be conservative since its existence has no impact on the usual interpretation of all other connectives. ${ }^{15}$ The same is true for co-intuitionistic logic (as structure of refutation), as can easily be checked.

Now, since we already have direct refutation in the co-intuitionistic logic of refutation, we have that the refutation of $\alpha$ is a solution (and this is monotonic in the sense that, when $\alpha$ is refuted, it remains so), whilst $\neg_{I} \alpha$ corresponds to the assumption of $\alpha$ leading to a non-proof, and so returns an "unsolved" problem (and this is non-monotonic); and $\neg_{C} \alpha$ corresponds to the assumption of $\alpha$ leading to a "non-refutation", so similarly returning an "unsolved" problem. To clarify this, let us now consider the relation between the two logics for proofs and refutations.

\section{Interpreting Problems and Solutions}

On the above, we have a co-constructive logic for proofs and refutations, the individual structures of which contain "weak" negations capable of expressing "non-proved" and "non-refuted", respectively, in addition to a mapping $(-)^{d}$. The latter is an involution which is permissible at any time, and corresponds to a switching of approach between "prover" and "refuter". In other words, on the level of problems, we are permitted, at any stage by means of $(-)^{d}$ to change our reference frame from approaching a formula from the point of view of attempting to prove it, or refute it. Moreover, whilst this mapping is involutive, it does not collapse to classical negation for two reasons. First, we understand $(-)^{d}$ to be an involution without fixed points, and second, we have syntactically separated the language. The latter means that certain "formulas" are barred in the sense that they can not be "well formed". For example, $\neg_{I} \alpha^{d}$ and $\neg_{C} \alpha$ are not wff 's, and so neither is $\neg_{I} \neg_{C} \alpha$. So, the two structures, whilst allowed to communicate via $(-)^{d}$, are also kept separate. Otherwise, as is known, the combined structure will collapse to a single partial order. ${ }^{16}$

As should be obvious, then, the mapping $(-)^{d}$, whilst it obviously has many of the properties of the "strong negation" discussed above, can not be identified with it. Rather, it will be the case that, only under certain circumstances, $(-)^{d}$ plays the role of strong negation. In fact, as we shall see, the circumstances in which this is possible corresponds exactly to those in which are dealing with propositions, or solutions, rather than problems. In this sense, strong negation operates metalinguistically as a way of demarcating a fundamental relation of preclusivity between a direct proof and a direct refutation. Let us consider this is more detail. 
The weak negations may be interpreted in standard Kripke-interpretations as: $s_{1} \|{ }^{+} \neg_{I} \alpha$ when " $\alpha$ is not amongst the set of proved statements at stage $s_{1}$ "; and, $s_{1} \|-{ }^{-} \neg_{C} \alpha$ as " $\alpha$ is not amongst the set of refuted statements at stage $s_{1}$ ". There are, therefore, four "modes" of proof and refutation that are characterizable in the structure as a whole:

$$
\begin{gathered}
\mid{ }_{I} \alpha \leftrightarrow \text { Direct proof of } \alpha \\
{ }_{C}-\alpha \leftrightarrow \text { Direct refutation of } \alpha^{d} \\
\left.\alpha\right|_{I} \leftrightarrow \text { Indirect proof that } \alpha \text { is unproved } \\
\alpha_{C}-\leftrightarrow \text { Indirect refutation that } \alpha^{d} \text { is unrefuted }
\end{gathered}
$$

The relationship between direct and indirect potential proofs (refutations) can be represented by the following diagram, ( $\alpha_{u p}$ indicates that $\alpha$ is not proved; $\alpha_{u r}^{d}$ that $\alpha^{d}$ is unrefuted):

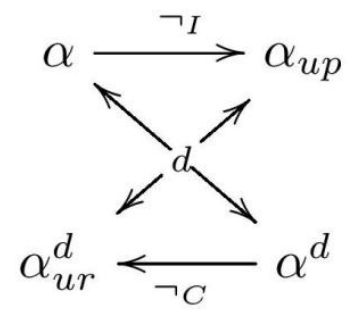

Remark 3. The resultant structures for $I$ and $C$ can now be better understood in relation to the properties of paracompleteness and paraconsistency, respectively. Due to the way in which we have constructed the two, it is not the case that law of non-contradiction is refutable in $I$, nor is law of excluded middle provable in $C$. Rather, there exist only indirect proofs (refutations) that they are unproved (unrefuted). Furthermore, the non-derivability of law of excluded middle in I is exactly mirrored by the non-derivability of law of non-contradiction in $C$ :

$$
\frac{\frac{\overline{\alpha \mid-{ }_{I} \alpha}(\text { Axiom })}{\mid-{ }_{I} \alpha, \neg_{I} \alpha}\left(\neg_{I} R\right)}{\frac{\mid-{ }_{I} \alpha, \alpha \vee \neg_{I} \alpha}{\mid-{ }_{I} \alpha \vee \neg_{I} \alpha, \alpha \vee \neg_{I} \alpha}(\vee R)}(\vee
$$$$
\frac{\frac{\frac{\alpha{ }_{C}-\alpha}{{ }_{C}-\mid \alpha, \neg_{C} \alpha}\left(\neg_{C} R\right)}{{ }_{C}-\alpha, \alpha \wedge \neg_{C} \alpha}(\wedge R)}{{ }_{C}-\alpha \wedge \neg_{C} \alpha, \alpha \wedge \neg_{C} \alpha}(\wedge R)
$$

In both cases, non-derivability results from the prohibition of contraction on the right of $\mid{ }_{I}$ and ${ }_{C}^{-}-$. Moreover, just as $\left.\neg_{I}\left(\alpha \vee \neg_{I} \alpha\right)\right|_{I}$ is derivable intuitionistically, so too is $\neg_{C}\left(\alpha \wedge \neg_{C} \alpha\right){ }_{C}-$ co-intuitionistically.

Now, consider again the distinction between problems and solutions. It is natural, in this setting to take a conclusive proof (refutation) to mark the end-point of a questioning process: "is $\alpha$ provable?", "is $\alpha$ refutable?". A “solution", to borrow Kolmogorov's terminology, is a determinate and conclusive answer to these questions. Of course, a problem can not be true nor false, and neither can it be asserted nor denied. For a proposition, $\alpha$, however, we should be able to say of $\alpha$ 
that it "holds", that $\alpha$ is true (false), and so on. We can think of a proposition $\bar{\alpha}$ as the result, or solution, to a problem, which terminates attempts to answer that problem. Then, the mapping $\overline{(-)}$ : $\alpha \rightarrow \bar{\alpha}$ and $\alpha^{d} \rightarrow \overline{\alpha^{d}}$ will denote that we have moved from consideration of $\alpha$ as a problem to a conclusive solution of that problem.

We note two features of conclusive proofs (refutations). First, conclusive proofs (refutations) will be constructive since it is not possible to destroy a conclusive proof (refutation) at any later stage of reasoning. Second, the relation between conclusive proofs and conclusive refutations is one of preclusivity, since it is not possible for their to exist a refutation of $\alpha$ whenever there exists a solution which says that $\alpha$ is proved. It is, therefore, at this point that we can allow $I$ and $C$ to directly communicate, such that for some $\alpha$, we have that either $\varnothing \mid-\alpha$ or $\varnothing-\alpha^{d}$, and $(\varnothing \mid-\alpha) \cap\left(\varnothing-\mid \alpha^{d}\right)=\varnothing$. refutations.

To clarify, let us briefly define a partial Kripke-structure for conclusive proofs and

Definition 8. Define a (partial) Kripke-structure for conclusive proofs and refutations as the combined structure $\mathrm{M}={ }_{d f}\left(\mathrm{~S}, \leq_{+}, \leq_{-}, V^{+}, V^{-}\right)$. We let $\mid=^{+}$and $\left.\right|^{-}$denote constructive forcing relations over conclusive proof and refutation, respectively. Then, at any stage, $s_{i}$, we define: $V^{+}(\alpha)=\left\{\alpha \in S: s_{i}^{+} \alpha\right\}$ and $V^{-}(\alpha)=\left\{\alpha \in S: s_{i}^{-} \alpha\right\}$. Furthermore, $\left.\right|^{+}$and $\left.\right|^{-}$are constructive for both $V^{+}$and $V^{-}$, satisfying the following persistence properties:

If $s_{1} \leq_{+} s_{2}$ then $\forall \alpha \in\left(V^{+}, s_{1}\right), \alpha \in\left(V^{+}, s_{2}\right)$; and If $s_{1} \leq_{-} s_{2}$ then $\forall \alpha \in\left(V^{-}, s_{1}\right), \alpha \in\left(V^{-}, s_{2}\right)$.

This ensures that, whenever a formula is conclusive assertible (deniable) at a stage, that "state" remains at every stage upstream, so, if $s_{1} \mid=^{+} \alpha$, then $s_{2} \mid=^{+} \alpha$; if $s_{1} \mid=^{-} \alpha$, then $s_{2} \mid={ }^{-} \alpha$.

Theorem 3. $\leq_{+}$and $\leq_{-}$are partial orders, which are reflexive, monotonic and transitive.

Proof. Obvious.

The clauses defining compound formulas are relatively standard (see e.g. [20]), but unlike standard Kripke-structures, there is no clause for negation since that can only ever be involved in an indirect proof (refutation). ${ }^{17}$ refutations):

$$
\begin{gathered}
{[\wedge] s_{i} \mid=^{+}(\alpha \wedge \beta) \text { iff } \alpha \in\left(V^{+}, s_{i}\right) \text { and } \beta \in\left(V^{+}, s_{i}\right)} \\
{\left.[\wedge] s_{i}\right|^{-}(\alpha \wedge \beta) \text { iff } \alpha \in\left(V^{-}, s_{i}\right) \text { or } \beta \in\left(V^{-}, s_{i}\right)} \\
{[\vee] s_{i} \mid=^{+}(\alpha \vee \beta) \text { iff } \alpha \in\left(V^{+}, s_{i}\right) \text { or } \beta \in\left(V^{+}, s_{i}\right)} \\
{[\vee] s_{i} \mid={ }^{-}(\alpha \vee \beta) \text { iff } \alpha \in\left(V^{-}, s_{i}\right) \text { and } \beta \in\left(V^{-}, s_{i}\right)}
\end{gathered}
$$




$$
\begin{aligned}
& {[\Rightarrow] s_{i} \mid=^{+}(\alpha \Rightarrow \beta) \text { iff } \forall s_{i}^{\prime} \text { and } s_{i} \leq s_{i}^{\prime}, \alpha \notin\left(V^{+}, s_{i}^{\prime}\right) \text { or } \beta \in\left(V^{+}, s_{i}^{\prime}\right)} \\
& {[\Leftarrow] s_{i} \mid=^{-}(\alpha \Leftarrow \beta) \text { iff } \forall s_{i}^{\prime} \text { and } s_{i} \leq s_{i}^{\prime}, \alpha \in\left(V^{-}, s_{i}^{\prime}\right) \text { or } \beta \notin\left(V^{-}, s_{i}^{\prime}\right)}
\end{aligned}
$$

We also note the following restriction on these structures, given preclusivity:

\section{Definition 10 (Restrictions on Kripke-structures)}

$$
\begin{array}{ll}
\forall s_{i} \in S, \alpha \in\left(V^{+}, s_{i}\right) \leftrightarrow & \alpha \notin\left(V^{-}, s_{i}\right) \\
\forall s_{i} \in S, \alpha \in\left(V^{-}, s_{i}\right) \leftrightarrow & \alpha \notin\left(V^{+}, s_{i}\right)
\end{array}
$$

Moreover, we have a metalinguistic device of a "strong" negation at the level of solutions. So, for example, we can say that, for any semantic interpretation of solutions, and any "solved" proposition $\alpha$, that either $\alpha \in V^{+}$, or $\alpha \in V^{-}$, and, for no $\alpha$ is $\alpha \in V^{+}$and $\alpha \in V^{-}$. If we think of this in terms of the development of constructive theories over time, then we have the relatively simple procedure of just "adding" a solution to the appropriate set of sentences that are either proved or refuted by that stage in the development of the theory. The discrepancy between typical construals of negation, then, is that "strong" negation is applicable only to solutions, whilst there also exist two, dual, weak negation operators that are applicable only to problems. Such a demarcation may not be to everybody's taste, but it allows us to capture what is constructive about constructive logic.

It is simple to give an algebraic semantics for solutions in terms of the characteristic functions of the set of proved and refuted sentences.

Definition 11 (Partial semantic structure). Define a partial semantic structure as an ordered pair $\langle S, V\rangle$, where $S$ is an enumerable set of formulas, and $V$ is a valuation space representing a structure of admissible valuations on the language. ${ }^{18}$ Then, let, $\mathrm{V}=\{1,0\}$ be a set of truth-values, and $\mathrm{D}=\{1\}$ be a proper subset of $\mathrm{V}$ which is a designated value. A valuation $v$ is a partial function on $S$ assigning a truth-value $\in \mathrm{V}$ to a formula $\alpha \in S$, where $v: S \rightarrow\{\mathrm{V}\}$.

The valuation function is extended by induction on the complexity of the Kripke clauses given above. Then, if we let $\Gamma^{\prime}$ denote the set of propositions which are proved at a stage $s$, and $\Delta^{\prime}$ the set of propositions that are refuted at $s$ :

Definition 12 (Characteristic function). For any $\left\langle\Gamma^{\prime}, \Delta^{\prime}\right\rangle$ we take a valuation to be the characteristic function such that:

$$
v\left(\Gamma^{\prime}\right)=\left\{v: v(\alpha)=1 \text { for each } \alpha \in \Gamma^{\prime}\right\} ; \text { and } v\left(\Delta^{\prime}\right)=\left\{v: v\left(\alpha_{i}\right)=0 \text { for each } \alpha_{i} \in \Delta^{\prime}\right\} .
$$

Lemma 2. We define a valuation $v$ over formulas $\alpha \in S$ as $\{v: \alpha \rightarrow\{1,0\}\}$, $v(\alpha)=1 \leftrightarrow \varnothing \mid-_{I} \alpha, \quad v(\alpha)=0 \leftrightarrow \varnothing_{C}-\alpha$. Then, $\langle S, V\rangle$ is simply the collection of all such functions.

The resultant semantic structure is obviously sound and complete with respect to the solutions of the combined structure. In fact, since we can think of each stage of the construction as constituting a partial interpretation where the formulas of $\Gamma_{i}$ are mapped to the valuation $\{1\}$ in a 
semantic structure, and the formulas in $\Delta_{i}$ to $\{0\}$, we have that, for every atomic $\alpha$, which is a solution;

$$
\text { Either } \exists \Gamma^{\prime}\left(\Gamma^{\prime} \mid{ }_{I} \alpha\right) \text { or, } \exists \Delta^{\prime}\left(\Delta^{\prime}{ }_{C}-\alpha\right) \text {. }
$$

\section{1. "Not-yet" proved or refuted}

What should we say, then, regarding problems that are not yet solved? There, we have problems which are not-yet actualized as solutions. This brings with it an obvious relation with the above distinction between actualism and potentialism. In effect, it is possible to understand problems as a space of potential proofs (refutations), and solutions as actual proofs (refutations). Moreover, we might also view the syntactical operations of co-constructive logics as properly lying "beneath" what is, in essence, a bivalent determination of propositions. Since, if, for example, we allow propositions to be identified with their proofs (refutations) in a simple extension of Brouwer's position, then bivalence holds for all propositions as is simple to see from the above semantic interpretation of solutions. This carries over from a translation of Dummett's argument that no statement may be both verified and falsified, together with the fact that a decision procedure holds for every solution. We can put this as a principle as follows:

Coherence: For no proposition $\bar{\alpha}$ is it the case that $\alpha \in V^{+}$, and $\alpha \in V^{-}$; and for each $\bar{\alpha}$, either $\alpha \in V^{+}$or $\alpha \in V^{-}$.

This much is standard, and, if it were to hold unrestrictedly for all formulas, brings with it a form of classical logic. What is important, however, from the point of view of constructivism, is that coherence does not hold across the board. Rather, our co-constructive logic allows us to deal, in addition, with states that are "paracoherent"

Paracoherence: Coherence holds, restrictedly, for solutions, whilst otherwise the structure of proofs and refutations (for problems) may be non-trivially undetermined and inconsistent.

In fact, I would go so far as to suggest that this captures the raison d'ętre of constructive logic.

\section{References}

1. Bellin, G., Carrara, M., Chiffi, D., and Menti, A. Pragmatic and dialogic interpretations of biintuitionism. Logic and Logical Philosophy, 2014.

2. Brouwer, L. L.E.J. Brouwer: collected works. Amsterdam: North-Holland Publishing Company, 1975.

3. Cook, R. T. and Cogburn, J. What negation is not: Intuitionism and ' $0=1$ ', Analysis, 60(265):512, 2000.

4. Crolard, T. Subtractive logic, Theoretical Computer Science, 254(1):151-185, 2001.

5. Dubucs, J. Feasibility in logic, Synthese, 132(3):213-237, 2002.

6. Dubucs, J. and Marion, M. Radical anti-realism and substructural logicsm, [in:] Rojszczak, A., Cachro, J., and Kurczewski, G. (editors), Philosophical Dimensions of Logic and Science, pages 235-249. Kluwer Academic Publishers, 2003.

7. Dummett, M. Reply to Dag Prawitz, [in:] Taylor, B. (editor), Michael Dummett: Contributions 
to Philosophy, pages 281-316. Distributors for the United States and Canada: Kluwer Academic Publishers, 1987.

8. Dummett, M. A. E. The seas of language. Oxford: Oxford University Press, 1993.

9. Dummett, M. A. E. Elements of intuitionism. Oxford: Oxford University Press, 2000.

10. Dunn, J. M. and Hardegree, G. Algebraic methods in philosophical logic. Oxford: Oxford University Press, 2001.

11. Goodman, N. The logic of contradictions, Zeitschrift fur Mathematische Logic und Grundlagen der Arithmetik, 27:119-126, 1981.

12. Hardegree, G. M. Completeness and super-valuations, Journal of Philosophical Logic, 34(1):81-95, 2005.

13. Heyting, A. Intuitionism. Amsterdam: North-Holland Pub. Co., 1971.

14. Kolmogorov, A. N. Zur Deutung der Intuitionistischen Logic, Mathematische Zeitschrift, 35:58-65, 1932.

15. Mancosu, P. From Brouwer to Hilbert: The debate on the foundations of mathematics in the 1920s. Oxford: Oxford University Press, 1998.

16. Martin-Löf, P. On the meanings of the logical constants and the justifications of the logical laws, Nordic Journal of Philosophical Logic, 1(1):11-60, 1996.

17. Martino, E. and Usberti, G. Temporal and atemporal truth in intuitionistic mathematics, Topoi, 13(2):83-92, 1994.

18. Mortensen, C. Inconsistent mathematics, Vol. 312. Springer, 1995.

19. Nelson, D. Constructible falsity, Journal of Symbolic Logic, 14(1):16-26, 1949.

20. Pietz, A. Not quite intuitionism, 2011. http://epub.ub.uni-muenchen.de/12589/1/DGPhil_Pietz.pdf

21. Prawitz, D. Meaning approached via proofs, Synthese, 148(3):507-524, 2006.

22. Priest, G. Dualising intuitionictic negation, Principia, 13(2):165-184, 2009.

23. Raatikainen, P. Conceptions of truth in intuitionism, History and Philosophy of Logic, 25(2):131-145, 2004.

24. Rauszer, C. Applications of Kripke models to Heyting-Brouwer logic, Studia Logica, 36(12):61-71, 1977.

25. Ripley, D. Embedding denial, [in:] Caret, C., and Hjortland, O., (eds.), Foundations of Logical Consequence, Oxford University Press, forthcoming 2015.

26. Shramko, Y. Dual intuitionistic logic and a variety of negations: The logic of scientific research, Studia Logica, 80(2-3):347-367, 2005.

27. Shramko, Y., Dunn, J. M., and Takenaka, T. The trilaticce of constructive truth values, Journal of Logic and Computation, 11(1):761-788, 2001.

28. Skura, T. On pure refutation formulations of sentential logics, Bulletin of the Section of Logic, 19(3):102-107, 1990.

29. Słupecki, J., Bryll, G., and Wybraniec-Skardowska, U. Theory of rejected propositions. I, Studia Logica, 29(1):75-123, 1971.

30. Słupecki, J., Bryll, G., and Wybraniec-Skardowska, U. The theory of rejected propositions. II, Studia Logica, 30(1):97-145, 1972.

31. Solomon, G. and DeVidi, D. Empirical negation in intuitionistic logic, [in:] DeVidi, D., Kenyon, T. (eds.) A logical Approach to Philosophy, pages 151-168. Springer, 2006.

32. Tennant, N. Negation, absurdity and contrariety, [in:] Gabbay, D. M., Wansing, H., (eds.) What is Negation?, pages 199-222. Springer, 1999.

33. Trafford, J. Abstract duality and co-constructive logics, manuscript under submission, 2014.

34. Trafford, J. Duality and Inferential Semantics, Axiomathes, forthcoming 2015.

35. Urbas, I. Dual-intuitionistic logic, Notre Dame Journal of Formal Logic, 37(3):440-451, 1996. 
1. To get ahead of ourselves, this property is mirrored in co-intuitionistic logic by the "conjunction property", where $-\alpha \wedge \beta$ iff $-\mid \alpha$ or $-\mid \beta$, and, since we understand co-intuitionism constructively, the disjunction property can not be central to a definition of constructivity.

2. I will not enter into a historical exegesis regarding the two positions (on this, the references above are more than adequate).

3. "In addition to theoretical logic, which systematizes a proof schemata for theoretical truths, one can systematize a proof schemata for solutions to problems [...] In the second section, assuming the basic intuitionistic principles, intuitionistic logic is subjected to a critical study; it is thus shown that it must be replaced by the calculus of problems, since its objects are in reality problems, rather than theoretical propositions" [13, p.58] (Translated in [15]).

4. See also Martino and Usberti [17]:

From the fact that possibility is conceived as tenseless it follows that the following principle of Potential Excluded Middle: (PEM) A is potentially true or A is not potentially true becomes intelligible, and valid, in its classical reading. For, on this reading, it simply means that all propositions, as they are conceived by the potential intuitionist, are atemporally determinate, and this is clearly true: if it were indeterminate whether A is provable or not, the provability of A would be for ever prevented since, according to the conception at issue, a proposition cannot become provable. Therefore, such a hypothetical state of indeterminateness of A could be nothing but a state of well-determined unprovability of A. Whether A is provable or not is a fact concerning the immutable world of propositions, where there is no room for any indeterminateness.

5. Though formulas of $S^{d}$ will typically be decorated with superscript in what follows (e.g. $\alpha^{d}$ ) unless context is obvious.

6. Co-implication is sometimes called "subtraction", e.g. [11], and typically, " $\alpha \leftarrow \beta$ " is read as " $\alpha$ without $\beta$ ", though this is not the case in our refutation interpretation. Further discussion can be found in [24], [35].

7. Obviously, we are working with a formal language here, so we probably don't strictly need to use the phrase "wellformed formula" (wff) of L. I should flag up that I interpret the phrase quite liberally, allowing, for example " $\{\phi \mid$ ... $\}$ " to be a wff.

8. See [34] for a discussion of the duality of classical logic in the context of constructing an inferentialist semantics.

9. This can be made formally precise through the notion of a Galois connection between two structures as explored in [33].

10. There are several issues regarding the interpretation discussed in [17, §11], that our interpretation in terms of refutation may solve, though this requires further investigation.

11. This is close to the view suggested in [35].

12. Note that this is a syntactic term, which must be distinguished from the semantic "indeterminate" of logics such as $K_{3}$.

13. One way around this (that I will not follow up here) might be to weaken the conditions on derivation of a contradiction, so that, for example, following [22], we think of $\perp$ as expressing something that is antithetical to the inquiry that we are interested in. So, we could think of $\perp$ as some theory that has no proof, but is much weaker than an outright contradiction. Then, any proof of $\alpha \Rightarrow \perp$ shows, indirectly that, $\alpha$ is antithetical to our inquiry at that stage. In other words, it allows us to express that, at that specific stage of reasoning, $\alpha$ is unprovable. Depending upon how this is cashed out, this would not necessarily preclude the existence of a proof of $\alpha$ at some later stage, in which, for example, our assumptions or data have changed such that $\alpha$ no longer leads us to a theory antithetical to inquiry.

14. This is close to Tennant's [32] conjecture (though clearly divergent from his conclusions), that negation may be understood as a constraint upon the space of possible proofs in terms of contrariness.

15. Though, this is not the route that I suggest.

16. If, for example, we relaxed the separability condition so that both negation operators may be applied to any formula, so that, $\neg_{I} \alpha^{\perp}$, and $\neg_{C} \alpha$ are $w f f$ 's, then an application of both negations to a formula, (e.g. $\neg_{I} \neg_{C} \alpha$ ), is obviously equivalent to Boolean negation. See also [4], [1].

17. We could, of course, add our "strong" negation along the lines of (3) and (4) given above, but I do not wish to confuse matters.

18. See e.g. [10], [12]. 
19. I borrow this term from [25], where it used in a rather different context but is conducive to this understanding: "a paraconsistent approach amounts to a paracoherent approach, in which one can be locally incoherent without global incoherence [...] Taking the paracoherentist option I'm suggesting here amounts to choosing to maintain a certain amount of reflective tension." 